\title{
Preparation of Nanocellulose from Cotton Fibers in Deep Eutectic Solvent (DES) and its Application in Paper
}

\begin{abstract}
Xiaonan Deng, ${ }^{\mathrm{a}}$ Lingzhong Wan, ${ }^{\mathrm{a}}$ Hui Sun, ${ }^{\mathrm{a}}$ Changfeng Li, ${ }^{\mathrm{a}}$ Fangzhi Liu,
XiaomingYan, ${ }^{a}$ Kun Liu, ${ }^{\text {b, } *}$ and Sihong $\mathrm{Ye}^{\mathrm{a}, *}$

The bond strength and stability of the intermolecular network structure of cellulose play a decisive role in the tensile strength and rupture resistance of paper. In this work, the surface morphology, the chemical composition, and the crystal structure of nanocellulose prepared from cotton fibers were characterized by scanning electron microscopy (SEM) analysis, X-ray diffraction (XRD), and Fourier transform infrared spectroscopy (FTIR) to prepare cotton fiber nanocellulose by green solvent deep-eutectic solvent pretreatment combined with the ultrasonic method. Nanocellulose of different morphology and structure was used as a papermaking additive to explore its effect on the strength performance of the paper. The results show that the burst resistance index of the paper handsheets could be increased by $6.1 \%$ and $9.8 \%$. The burst resistance index improved as the added amount of the NFC was increased.
\end{abstract}

DOI: 10.15376/biores.17.1.714-724

Keywords: Deep eutectic solvent; Cotton Nanocellulose Fiber; Papermaking

Contact information: a: Cotton Research Institute of Anhui Academy of Agricultural Sciences, 40 Nongke Road, Hefei, Anhui, China, 230001; b: Department of Chemical Engineering and Technology, College of Chemistry and Chemical Engineering, Hefei University of Technology, 193 Tunxi Road, Hefei, Anhui, China, 230009; *Corresponding author: dengxn6235@qq.com

\section{INTRODUCTION}

Paper is generally made from plant-derived materials, so its chemical composition is mainly comprised of cellulose, hemicellulose, and lignin. The microscopic morphology reveals that the diameter of the plant fibers is on the micrometer to millimeter level. The fibers are intertwined and woven into the network structure, so there is a large amount of hydrogen bonding to impact mechanical strength in the paper. When cellulose is converted to a nanofibrillar structure, it has excellent properties such as high mechanical strength, good thermal stability, large specific surface area, high transmittance, low density, and renewability, among others (Feng et al. 2018; Lu et al. 2019). Micro/nano fibrillated cellulose (MNFC), which also has been called cellulose nanofibril, can be used in the enhancement of biological composite materials and in the preparation of hydrogels, electronic devices, and papermaking auxiliaries. Many studies have reported the application of nanofibrillated cellulose (NFC) in the paper industry. For example, NFC has been used as an additive to improve the physical properties of paper (tensile strength, barrier properties, or the production of smart packaging paper) (Xiang et al. 2016; Sirviö 2019).

As a new type of ionic liquid analog, deep eutectic solvents (DESs) have been widely used in extraction, reaction, material processing, and electrochemistry. The DESs can be regarded as analogs to ionic liquids. They have favorable characteristics such as low 
cost, simple preparation process, non-toxic nature, low vapor pressure, and biodegradability (Alonso et al. 2016). Meanwhile, their properties can be tailored by varying the components and composition to satisfy the requirements of specific applications (Smith et al. 2014; Khandelwal et al. 2016; Mbous et al. 2017; Zdanowicz et al. 2018). The formation of DES requires a hydrogen bond donor and a hydrogen bond acceptor. These are provided by a mixture of salt and a hydrogen bond acceptor or anions of a hydrogen bond donor and salt. The use of DES for the extraction of active ingredients in plant tissues, the pretreatment of wood raw materials, and the preparation and functionalization of nanocellulose has become a widely researched subject (Ling et al. 2018; Zdanowicz et al. 2018; Douard et al. 2021). The high efficiency refining of raw wood materials with DES as a green solvent and the development of new DESs have great potential for future research and development. Some scholars have reported that cotton dissolved in DES composed of choline chloride and oxalic acid can be used to prepare micro- and nano-sized cellulosic fibers. Oxalic acid can form carboxyl groups on the crystalline surface of cellulose fibers, resulting in filamentation of the fibers and splitting of crystal surfaces to form nanocellulose with low crystallinity and flakiness. Yu's group studied the efficient preparation of nanometer fiber in DES using microwave heating combined with the high intensity ultrasonic vibrations. They found that the treatment's effect on surface, morphology, and thermal stability of nanocellulose is similar to that of sulfuric acid treatment (Liu et al. 2017), while the processing cycle is significantly shortened. This work is expected to replace the traditional technology of preparing nanocrystalline cellulose by concentrated sulfuric acid hydrolysis and improve the industrialization of nanocrystalline cellulose preparation. However, all these latest studies do not provide information on the yield and application of nano-sized cotton fibers. Since the properties of DES vary with its combination of component and composition, new acidic natural DESs can be designed and synthesized for extraction of cellulose. In these extractions, the presence of acid and hydrogen bonding are helpful in breaking the original structure of the cellulose. Therefore, this study investigated the preparation of nano-sized cellulose from cotton fibers with a new DES.

In this study, ultrasonic waves combined with betaine/oxalic acid DES pretreatment were used to prepare nanofibrillated cellulose from cotton fibers. By optimizing the treatment time and the concentration, nanocellulose with a high yield was prepared. Scanning electron microscopy (SEM), X-ray diffraction (XRD), Fourier-transform infrared spectroscopy (FTIR), and thermogravimetric analysis (TGA) were used to characterize and test the properties of the prepared nanocellulose. Nanocellulose with different morphological and structural characteristics were used as paper auxiliaries and applied in the papermaking process to explore the influence of nanocellulose on the strength of paper.

\section{EXPERIMENTAL}

\section{Materials}

Cotton pulp fibers was obtained (Yinshan Cotton \& Linen Co., LTD). Oxalic acid dihydrate (purity: > 99\%) and betaine (purity: > 98\%) were obtained from Sinopharm Chemical Reagent Co., Ltd. (Shanghai, China). Sodium chloride (purity: > 99\%) was also obtained from Sinopharm Chemical Reagent Co., Ltd. (Shanghai, China). Deionized water was used throughout the experiment. The experiment cotton was Wankemian-1(Anhui Academy of Agricultural Sciences, Anqing, Anhui, China ( $30^{\circ} 31^{\prime}$ N, $117^{\circ} 06^{\prime}$ E).), a finite 
and infinite fruit branch variety as shown in Fig. 1.
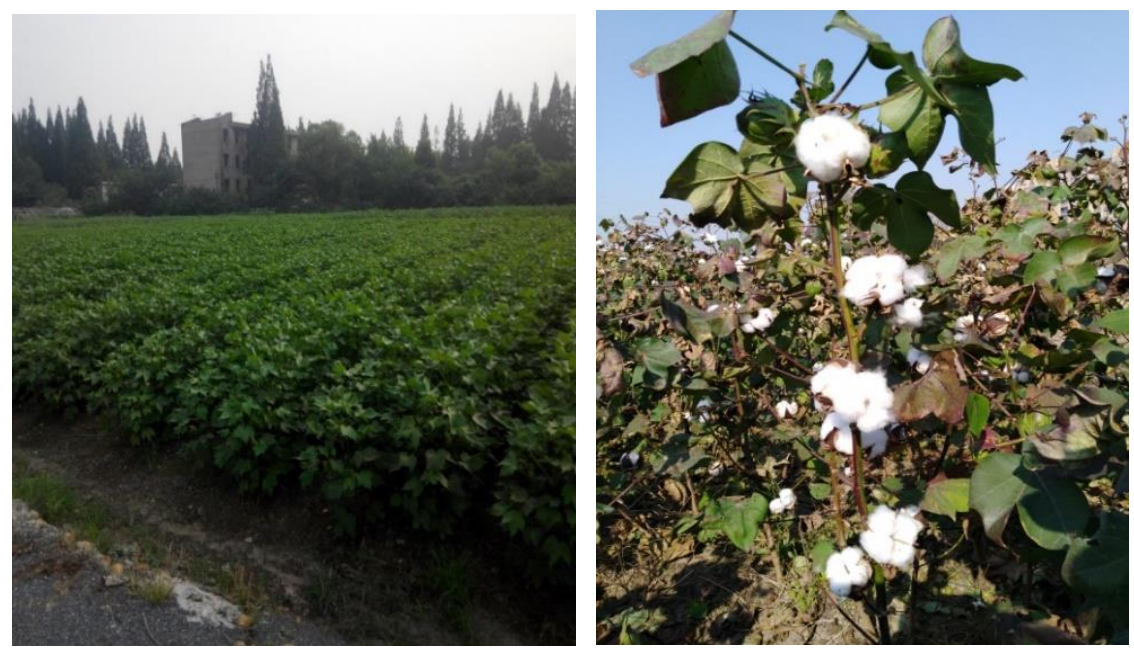

Fig. 1. The field experiment (cotton varieties Wankemian-1)

\section{Methods}

DES treatment

The 1:2 $\mathrm{M}$ ratio of acidic DES was produced by stirring $63.0 \mathrm{~g}$ of oxalic acid dihydrate and $69.8 \mathrm{~g}$ of betaine continuously for $30 \mathrm{~min}$ at $90{ }^{\circ} \mathrm{C}$ in a glass reactor. Cotton fibers weighing approximately $0.1 \mathrm{~g}$ were added to the reactor. At the end of the treatment, $200 \mathrm{~mL}$ of deionized water was added to the reactor to reduce the viscosity of the mixture. The treated cellulose was washed and filtered. The pretreated fibers were first mixed with water at a concentration of $0.5 \mathrm{wt} \%$. The suspensions were then processed using a JY99IIDN ultrasonic generator (Scientz Biotechnology Co., Ltd, Ningbo, China) equipped with a $25 \mathrm{~mm}$ cylindrical titanium alloy probe. The ultrasonication was conducted at a frequency of $20 \mathrm{kHz}$ with an output power $1000 \mathrm{~W}$ for $20 \mathrm{~min}$.

\section{Characterization and measurements.}

The chemical compositions of the cotton and the nanocellulose were determined. The morphology of the pretreated cotton fibers was observed using a JSM-7500F scanning electron microscope (SEM) (JEOL, Tokyo, Japan) at $15 \mathrm{kV}$. The samples were coated with platinum using a vacuum sputter coater before they were observed.

The FTIR spectra were recorded using a Nicolet Magna 560 FTIR instrument (Thermo Fisher Scientific Inc., Waltham, MA, USA) with a diamond attenuated total reflectance. The derivative thermogravimetry (DTG) analysis was performed using a Pyris6 TG analyzer (Perkin-Elmer, Waltham, MA, USA) at a heating rate of $10{ }^{\circ} \mathrm{C} \mathrm{min}{ }^{-1}$ under a nitrogen atmosphere.

In the paper application test, a range of length nanocellulose (50 to $100 \mu \mathrm{m})$ was poured into the disperser and dispersed for 5,000 revolutions. The mixed pulp was then placed in a manual paper making machine, which was made from a Rapid Köthen type laboratory sheet former (ISP mod. 786FH) according to ISO 5269-2. Handsheets were prepared and evaluated according to the standard method of TAPPI TIP0502-17 and T 205 sp-02. When operating the sheet-forming machine (RK3AKWT), the stainless-steel mesh or copper mesh is placed at the bottom of the molding cylinder, and the slurry and water are added. The slurry is evenly dispersed with the stirring paddle, and the vacuum lever is 
manually pulled to introduce vacuum and pull the water quickly from the paper quickly. The paper is removed from the wire mesh, and it is stored in the constant temperature and humidity room after drying.

The breaking index and the tensile strength were measured with a Lorentzen \& Wettre tensile strength meter (Stockholm, Sweden).

\section{Beating and paper handsheets forming}

About $30 \mathrm{~g}$ cotton pulp was diluted to $10.0 \mathrm{wt} \%$ by deionized water, and then beaten using a PFI mill (HAMJERN MASKIN 621) according to TAPPI method T248. The pulp was refined to $55^{\circ} \mathrm{SR}$. After that, the refined pulp was weighed and evenly dispersed in the pulp disintegrator for 10,000 revolutions. A selected amount of nanocellulose was added into the disintegrator for another 5,000 revolutions. The mixed slurry was used for preparing papers by a handsheet former (RK3AKWT).

The basis weight of the paper handsheets was $60 \mathrm{~g} / \mathrm{m}^{2}$. The control (no nanocellulose), the $2.5 \%$ cotton fiber nanocellulose, the $5 \%$ cotton fiber nanocellulose, the $2.5 \%$ wood fiber nanocellulose, and the $5 \%$ wood fiber nanocellulose handsheets were labeled as samples $\langle 1\rangle,\langle 2\rangle,\langle 3\rangle,\langle 4\rangle$, and $\langle 5\rangle$, respectively.

\section{Characterization and Measurements}

\section{Fiber analysis}

A $30 \mathrm{mg}$ oven-dry pulp sample was weighed and diluted in $1 \mathrm{~L}$ water so that the fibers were evenly dispersed in water. The dispersed slurry was placed in the instrument (Morfi Compact, Techpap) for testing.

\section{Burst index}

The tensile strength of the handsheets was measured according to TAPPI T 403 om-02. Each sample was placed between the upper and lower chuck of the tester. Care was taken that the sample exceeded the entire area of the upper chuck. The L\&W burst tester was started. The data were read after the breakage of specimens.

\section{Tensile index}

The tensile strength and whiteness of the handsheets were measured according to TAPPI T494 om-01 (2001) and TAPPI T562 pm-96 (1996), respectively. The sample was cut into a size of $15 \mathrm{~mm} \times 15 \mathrm{~cm}$, placed in the $\mathrm{L} \& \mathrm{~W}$ tensile strength meter for testing, and the display data was read.

\section{Optical properties}

The optical properties of the handsheets were measured according to ISO2470:1999. Ten layers of paper and five test points were used for each test. The color information of the specimens was quantified using the ColorTouch PC CTP-ISO; Technidyne, New Albany, IN, USA). The specific steps are as follows: Open the instrument test software Color Touch and select ERIC 950 in the File bar to enter the residual ink test interface. Then place a stack of samples (opaque) in the light path, select System Settings in the System bar to set test points before the test, and then press the Start button to Take Sample for the test. After that, place a single Sample on the black cylinder for the test and record the final result. In the File bar, select Color Brightness to test whiteness. The test steps are roughly the same as the operation steps. After the test, the black tube was placed back into the black plastic bag and power turned off. 


\section{RESULTS AND DISCUSSION}

\section{SEM Analysis for Nanofibrillated Cellulose by DES Pretreatment}

The surface morphology and size of the prepared cellulose nanocrystals were analyzed by SEM. Although the same ultrasound effects were used for all the samples, the size of the cellulose nanocrystals were not the same under different treatment conditions. At $90{ }^{\circ} \mathrm{C}$, approximately $100 \mu \mathrm{m}$ of sample were obtained. However, depolymerized micrometer-sized fragments of the cellulose nanocrystals were also observed. As shown in Fig. 2(a), nanocellulose was obtained at $100^{\circ} \mathrm{C}$. As the treatment temperature increased, the size of the cotton fibers decreased.

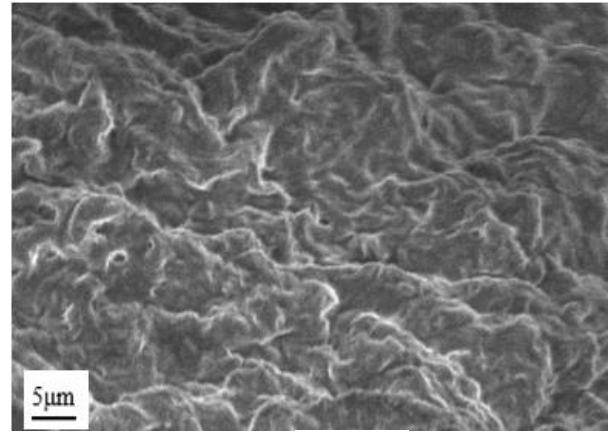

(a)

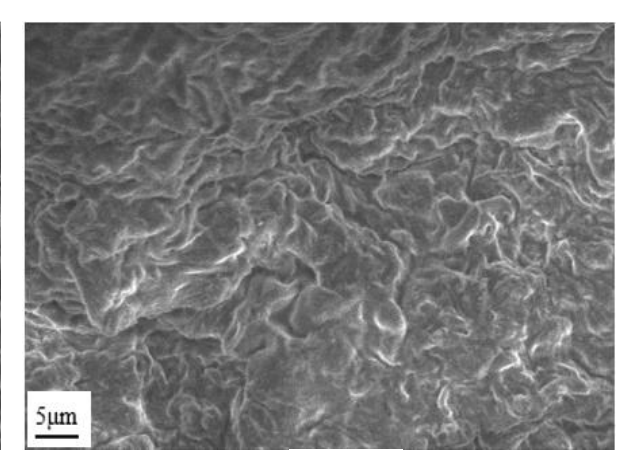

(b)

Fig. 2. SEM images of the pretreated cotton fibers by DES at a) $90{ }^{\circ} \mathrm{C}$ for 50 min and b) $100{ }^{\circ} \mathrm{C}$ for $50 \mathrm{~min}$

\section{TEM Analysis for Nanofibrillated Cellulose by DES Pretreatment}

TEM micrographs of the nanocellulose are shown in Fig. 3. The nanocellulose was prepared by eutectic solvent is a structure composed of cellulose fibrils with diameters of about $5 \mathrm{~nm}$. The temperature of deep eutectic solvent and subsequent mechanical treatment affect the dimensions of the resulting nanocellulose. Under relatively mild temperature conditions, there were still large fiber bundles, as shown in Fig. 3(a). The fibril diameters were in the range 100 to $200 \mathrm{~nm}$ and showed individual fibrils structure. Based on the appearance reveal in such micrographs, the authors judged that the nanocellulose could be classified as nanofibrillated cellulose (NFC).

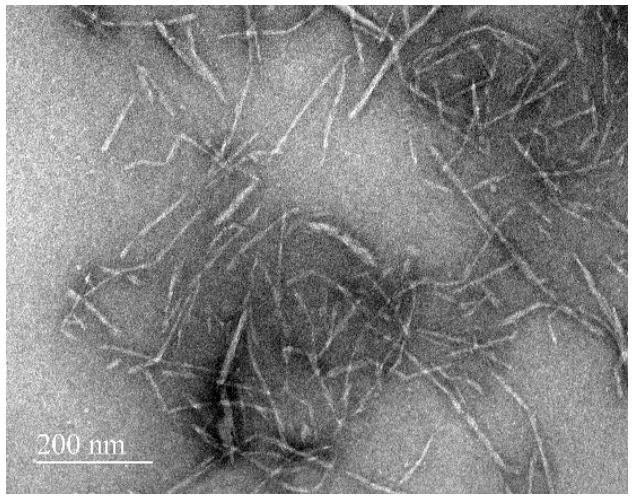

(a)

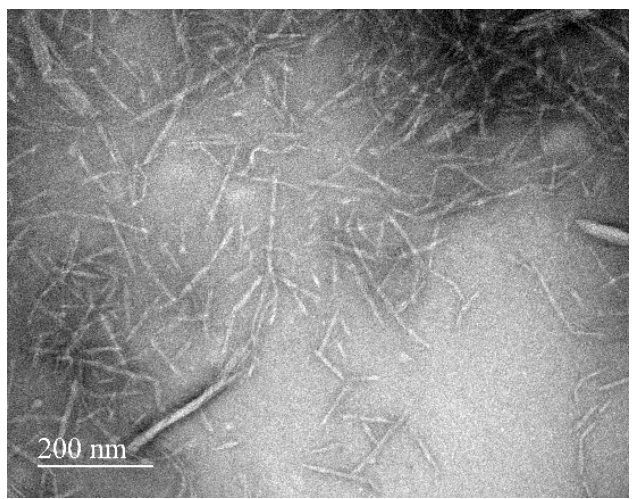

(b)

Fig. 3. TEM images of the products of pretreated cotton fibers by DES at a) $90^{\circ} \mathrm{C}$ for 50 min and b) $100^{\circ} \mathrm{C}$ for $50 \mathrm{~min}$ 


\section{Structural Analysis of the DES Pretreated Nanofibrillated Cellulose}

The structure of the fiber and nanocellulose pretreated with the DES was analyzed by FTIR to detect the changes in their surface groups compared to raw materials (Fig. 4). The characteristic absorbance peaks of the cellulose were 1431, 1376, 1165, 1117, 1062, and $899 \mathrm{~cm}^{-1}$. These peaks are consistent with the structure of cotton cellulose. In the infrared spectra of the pretreated fibers, a new peak appeared at $1726 \mathrm{~cm}^{-1}$, which indicated that the $\mathrm{C}=\mathrm{O}$ double bond was present on the surface of the pretreated fibers after the DES treatment. This may have been due to the carboxyl group on the surface of the pretreated fibers or the hemiacetal structure exposed by the degradation of the amorphous cellulose. The infrared spectra of the nanofibrillated cellulose were not different from those of the pretreated fibers, which indicated that they had similar chemical structures and groups. The purity of the prepared NFC was detected by elemental analysis. It can be seen from the results that the contents of the $\mathrm{C}, \mathrm{H}, \mathrm{N}$, and $\mathrm{S}$ in the pretreated fibers and NFC were similar to those of the raw cotton fiber.

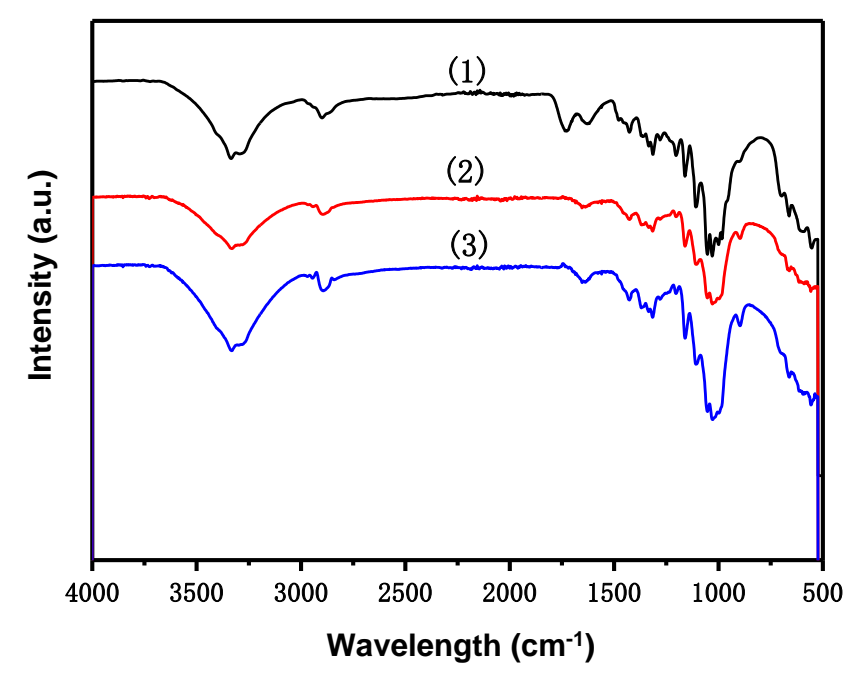

Fig. 4. FTIR spectra of the cotton fibers and the NFC

\section{Crystalline Morphology and Thermal Stability of the DES Pretreated Nanofibrillated cellulose}

The X-ray diffraction (XRD) method was used to study the crystal structure and relative crystallinity of the pretreated fiber and NFC. As shown in Fig. 5, the characteristic diffraction peaks of the raw cotton fiber, the pretreated cotton fiber, and the NFC at $2 \theta$ of $14.4^{\circ}, 16.3^{\circ}$, and $22.4^{\circ}$, indicated that their crystal structures were all cellulose type I. The results showed that the DES treatment did not change the crystal structure of the cellulose. Based on this finding it can be concluded that the DES did not act as a true solvent for the cellulose. As an important index that affects the mechanical properties and the thermal stability of cellulose, the crystallinity of the cotton fiber was calculated by the Segal method (Segal et al. 1959). The crystallinity of the cotton fiber was $61 \%$. After DES treatment, the crystallinity value of the cotton fiber increased by $5 \%$ to $20 \%$ compared to the raw material. The increase in crystallinity is apparently due to the dissolution of the heterogeneous primary cell wall and the removal of most of the oligosaccharides. 
The TG curve can be seen in Fig. 6. The initial degradation temperature of the cotton fiber was approximately $300{ }^{\circ} \mathrm{C}$, while the initial degradation temperature of the prepared nano fibrillated cellulose was raised to approximately $315{ }^{\circ} \mathrm{C}$. The maximum degradation rate temperature also showed a similar trend. The maximum degradation rate temperature of the NFC was $334^{\circ} \mathrm{C}$ and the maximum degradation rate temperature of the cotton fiber was $315^{\circ} \mathrm{C}$.

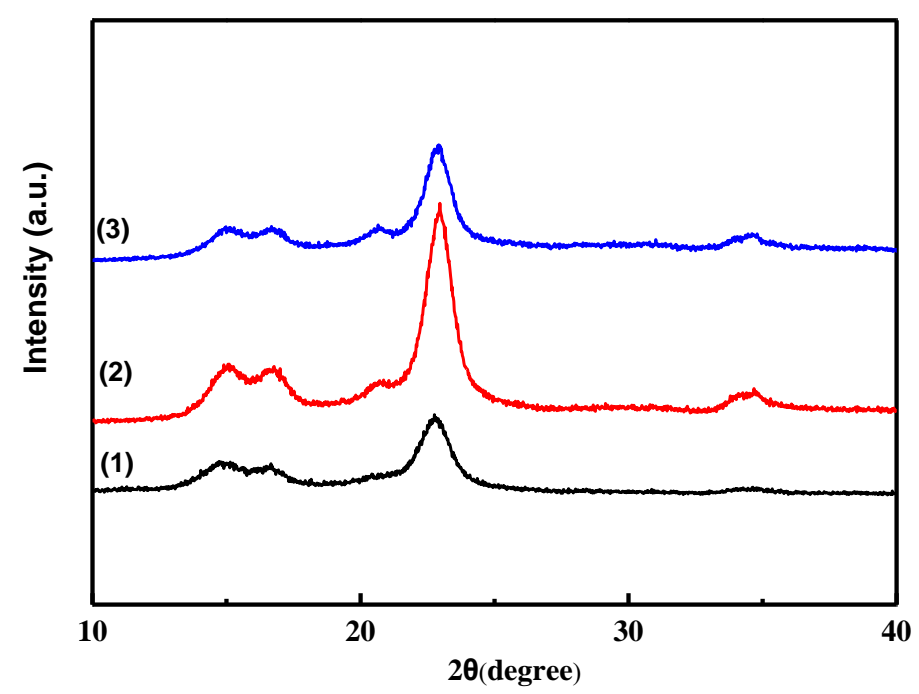

Fig. 5. XRD curves of the cotton fibers and the carbon of the modified cotton fibers

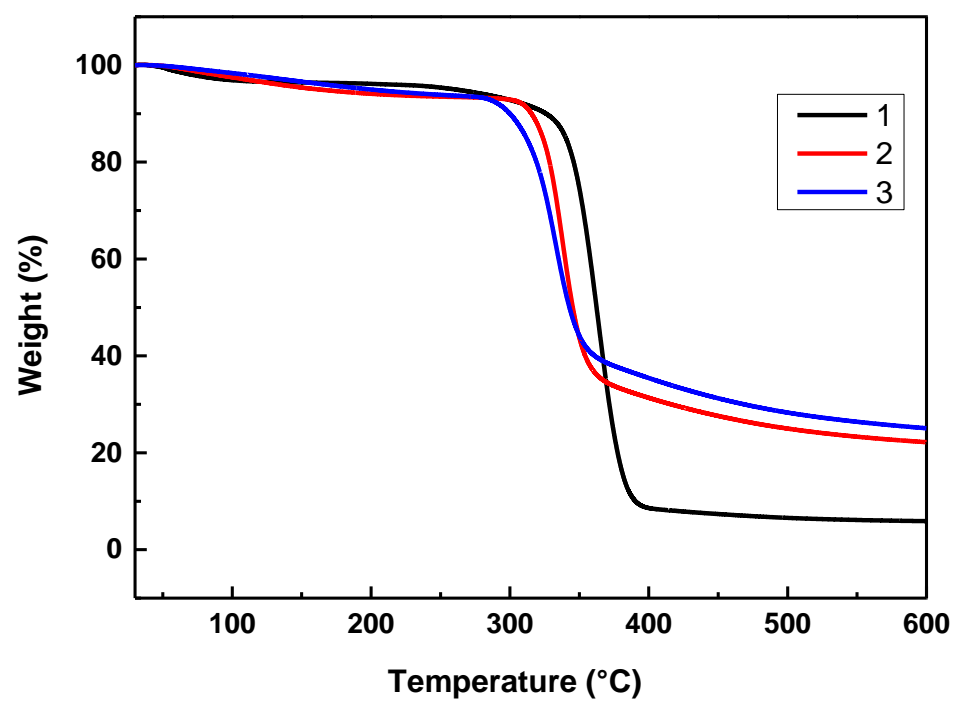

Fig. 6. The TG curves of the original cotton fibers and the modified cotton fibers

\section{Fiber Content Analysis}

The cotton fiber length, the fiber width, the curl index, the kink index, and the fine fiber content when the beating degree was $50{ }^{\circ} \mathrm{SR}$ are shown in Table 1. 
Table 1. Morphological Properties of Cotton Pulp Fibers

\begin{tabular}{|c|c|c|c|c|c|}
\hline Sample & $\begin{array}{c}\text { Length }(\mathbf{L}(\mathbf{n}), \\
\mathbf{m m})\end{array}$ & Width $(\boldsymbol{\mu m})$ & $\begin{array}{c}\text { Kink Index } \\
(\mathbf{})\end{array}$ & $\begin{array}{c}\text { Curl Index } \\
(\%)\end{array}$ & $\begin{array}{c}\text { Fine Fiber } \\
(\%)\end{array}$ \\
\hline $\begin{array}{c}\text { Cotton } \\
\text { Fiber }\end{array}$ & 0.752 & 18.5 & 137 & 14.8 & 2.95 \\
\hline
\end{tabular}

Tensile and bursting properties of handsheets are shown in Table 2. The average burst index of the paper handsheets without the nano fibrillated cellulose was 3.26 $\mathrm{kPa} \cdot \mathrm{m}^{2} / \mathrm{g}$, while the average breaking index values of the paper handsheets with $2.5 \%$ cotton fiber NFC and $2.5 \%$ wood fiber NFC were 3.58 and $3.46 \mathrm{kPa} \cdot \mathrm{m}^{2} / \mathrm{g}$, respectively. At the $2.5 \%$ nano fibrillated cellulose addition level, the burst resistance index of the paper handsheets increased by $9.8 \%$ and $6.1 \%$. Samples $\langle 3\rangle$ and $\langle 5\rangle$ showed that increasing the addition rate of the nano fibrillated cellulose, improved the burst resistance index.

Table 2. Burst Index of the Paper Handsheets

\begin{tabular}{|c|c|c|c|c|c|}
\hline \multirow{2}{*}{ Number } & \multicolumn{5}{|c|}{ Burst Index (kPa· $\left.\mathbf{m}^{2} / \mathbf{g}\right)$} \\
\cline { 2 - 6 } & $<1>$ & $<2>$ & $<3>$ & $<4>$ & $<5>$ \\
\hline Average & 3.26 & 3.58 & 3.74 & 3.46 & 3.54 \\
\hline
\end{tabular}

\section{Tensile Index}

As can be seen in Table 3, the strengthening trend of the tensile index for the paper handsheets with different additive amounts of cotton fiber NFC was consistent with the burst resistance index. There was little difference in the tensile index between the handsheets with $2.5 \%$ or $5 \%$ lignocellulosic NFC contents. This may be because the size of the lignin fiber NFC was too small for the paper-based fiber of this project, and a slight change in the addition amount was not enough to reflect the advantages of the enhancement.

Table 3. Tensile Index of the Paper Handsheets

\begin{tabular}{|c|c|c|c|c|c|}
\hline \multirow{2}{*}{ Number } & \multicolumn{5}{|c|}{ Tensile Index (N·m/g) } \\
\cline { 2 - 6 } & $<1>$ & $<2>$ & $<3>$ & $<4>$ & $<5>$ \\
\hline Average & 31.11 & 34.84 & 37.03 & 36.85 & 36.47 \\
\hline
\end{tabular}

\section{Optical Properties}

Table 4 shows that the optical properties of the paper handsheets did not change much with the $2.5 \%$ and $5 \%$ NFC additions, which was not much different from the default handsheets without NFC. Opacity refers to the opaque nature of the paper. The opacity of the paper depends on the light scattering ability of the paper, the number of light scattering interfaces inside the paper (the number of independent particles inside) and the difference in refractive index of the scattering interface. The more light scattering interfaces, the greater the refractive index difference between the scattering interfaces, the greater the light scattering ability of the paper, the higher the opacity. The addition of nanofibrillated cellulose has been reported to have little effect on optical properties (Tajik et al. 2016; Kasmani and Samariha 2019). 
Table 4. Optical Properties of the Paper Handsheets

\begin{tabular}{|c|c|c|c|c|c|c|}
\hline \multirow{2}{*}{ Number } & \multirow{2}{*}{ Whiteness } & \multirow{2}{*}{ Brightness } & \multicolumn{3}{|c|}{ Color Coordinates } & \multirow{2}{*}{ Opacity } \\
\cline { 4 - 6 } & & & $L^{*}$ & $a^{*}$ & $b^{*}$ & \\
\hline$<1>$ & 81.98 & 82.85 & 93.06 & -0.17 & 0.24 & 86.66 \\
\hline$<2>$ & 82.32 & 83.48 & 93.38 & -0.19 & 0.33 & 87.91 \\
\hline$<3>$ & 82.35 & 83.69 & 93.50 & -0.17 & 0.38 & 88.89 \\
\hline$<4>$ & 82.20 & 83.62 & 93.48 & -0.22 & 0.40 & 88.44 \\
\hline$<5>$ & 80.85 & 82.21 & 92.85 & -0.21 & 0.39 & 88.14 \\
\hline
\end{tabular}

\section{CONCLUSIONS}

1. This study demonstrated a facile and green method to produce qualified nanofibrillated cellulose (NFC) from cotton fiber. The NFC was incorporated into paper made from cotton fibers. The betaine/oxalic acid dihydrate deep eutectic solvent (DES) pretreatment system was found to be efficient for the cleavage of the strong hydrogen bonds in the cotton.

2. The deep eutectic solvent consisted of oxalic acid and betaine did not act as a true solvent for the cellulose When the addition of the nano fibrillated cellulose was $2.5 \%$, the burst resistance index and the tensile index of the handsheets increased by $9.8 \%$ and $6.1 \%$.

3. The presented method facilitates the green and fast processing and application of cotton fiber while not affecting the yield and properties of the nano fibrillated cellulose.

\section{ACKNOWLEDGMENTS}

The authors are grateful for the support of the Key Science and Technology Special Project of Anhui Province (Grant No. 202003b06020009). The authors also are grateful for the support of the Scientific Research Team Project of Anhui Academy of Agricultural Sciences (Grant No. 2021YL056).

\section{REFERENCES CITED}

Alonso, D. A., Baeza, A., Chinchilla, R., Guillena, G., Pastor, I. M., and Ramón, D. J. (2016). "Deep eutectic solvents: The organic reaction medium of the century," European Journal of Organic Chemistry 2016(4), 612-632. DOI:

10.1002/ejoc.201501197

Douard, L., Bras, J., Encinas, T., and Belgacem, M. N. (2021). "Natural acidic deep eutectic solvent to obtain cellulose nanocrystals using the design of experience approach," Carbohydrate Polymers 252, 117136. DOI:

10.1016/j.carbpol.2020.117136 
Feng, Y., Li, J., Wang, B., Tian, X., Chen, K., Zeng, J., Xu, J., and Gao, W. (2018). "Novel nanofibrillated cellulose/chitin whisker hybrid nanocomposites and their use for mechanical performance enhancements," BioResources 13(2), 3030-3044. DOI: 10.15376/biores.13.2.3030-3044

Kasmani, J., and Samariha, A. (2019). "Effect of nano-cellulose on the improvement of the properties of paper newspaper produced from chemi-mechanical pulping," BioResources 14(4), 8935-8949. DOI: 10.15376/biores.14.4.8935-8949.

Khandelwal, S., Tailor, Y. K., and Kumar, M. (2016). "Deep eutectic solvents (DESs) as eco-friendly and sustainable solvent/catalyst systems in organic transformations," Journal of Molecular Liquids 215, 345-386. DOI: 10.1016/j.molliq.2015.12.015

Ling, Z., Edwards, J. V., Guo, Z., Prevost, N. T., Nam, S., Wu, Q., French, A. D., and $\mathrm{Xu}, \mathrm{F}$. (2018). "Structural variations of cotton cellulose nanocrystals from deep eutectic solvent treatment: Micro and nano scale," Cellulose 26(2), 861-876. DOI: 10.1007/s10570-018-2092-9

Liu, Y., Guo, B., Xia, Q., Meng, J., Chen, W., Liu, S., Wang, Q., Liu, Y., Li, J., and Yu, H. (2017). "Efficient cleavage of strong hydrogen bonds in cotton by deep eutectic solvents and facile fabrication of cellulose nanocrystals in high yields," ACS Sustainable Chemistry \& Engineering 5(9), 7623-7631. DOI: 10.1021/acssuschemeng.7b00954

Lu, Z., An, X., Zhang, H., Guan, M., Liu, J., Sun, Y., Nie, S., Cao, H., Lu, B., and Liu, H. (2019). "Study on the wet-web strength and pressability of paper sheet during the press process with the addition of nano-fibrillated cellulose (NFC)," Carbohydrate Polymers 210, 332-338. DOI: 10.1016/j.carbpol.2019.01.083

Mbous, Y. P., Hayyan, M., Hayyan, A., Wong, W. F., Hashim, M. A., and Looi, C. Y. (2017). "Applications of deep eutectic solvents in biotechnology and bioengineeringPromises and challenges," Biotechnology Advances 35(2), 105-134. DOI: 10.1016/j.biotechadv.2016.11.006

Segal, L., Creely, J., Martin, A., and Conrado, C. (1959). “An empirical method for estimating the degree of crystallinity of native cellulose using the X-ray diffractometer," Textile Research Journal 29(10), 786-794. DOI: 10.1177/004051755902901003

Sirviö, J. A. (2019). "Fabrication of regenerated cellulose nanoparticles by mechanical disintegration of cellulose after dissolution and regeneration from a deep eutectic solvent," Journal of Materials Chemistry A 7(2), 755-763. DOI: 10.1039/C8TA09959F

Smith, E. L., Abbott, A. P., and Ryder, K. S. (2014). "Deep eutectic solvents (DESs) and their applications," Chemical Reviews 114(21), 11060-11082. DOI: $10.1021 / \mathrm{cr} 300162 \mathrm{p}$

Tajik, M., Resalati, H., Hamzeh, Y., Torshizi, H. J., Kermanian, H., and Kord, B. (2016). "Improving the properties of soda bagasse pulp by using cellulose nanofibers in the presence of cationic polyacrylamide," BioResources 11(4), 9126-9141. DOI: 10.15376/biores.11.4.9126-9141.

Xiang, Z., Gao, W., Chen, L., Lan, W., Zhu, J. Y., and Runge, T. (2016). “A comparison of cellulose nanofibrils produced from Cladophora glomerata algae and bleached eucalyptus pulp," Cellulose 23(1), 493-503. DOI: 10.1007/s10570-015-0840-7 
Zdanowicz, M., Wilpiszewska, K., and Spychaj, T. (2018). "Deep eutectic solvents for polysaccharides processing. A review," Carbohydrate Polymers 200, 361-380. DOI: 10.1016/j.carbpol.2018.07.078

Article submitted: July 23, 2021; Peer review completed: September 28, 2021; Revised version received: November 17, 2021; Second revision received and accepted: December 1, 2021; Published: December 7, 2021.

DOI: 10.15376/biores.17.1.714-724 